\title{
Advances in Tuberculosis: Therapeutics
}

\author{
Varinder Singh ${ }^{1} \cdot$ Sushil K. Kabra ${ }^{2}$
}

Received: 29 May 2019 / Accepted: 29 May 2019 / Published online: 18 June 2019

(C) Dr. K C Chaudhuri Foundation 2019

After discovery of streptomycin, significant improvement occurred over 2-3 decades in treatment of tuberculosis. Effective combination chemotherapy evolved over decades of research, thus dramatically reducing morbidity and mortality due to tuberculosis (TB). Further efforts led to development of modern day short term chemotherapy with high success rate and it appeared that perhaps elimination of TB will be a reality, however this euphoria was short lived. Declining burden in the developed nations saw a u-turn and this resurgence of TB was associated with increasing drug resistant TB. Emergence of human immunodeficiency virus (HIV) further boosted this reversal of trends. World Health authorities had to declare tuberculosis as an emergency in 1993. While the world grappled with rising trends of drug resistance $\mathrm{TB}$, the treatment success rates for multi drug resistant (MDR) TB were poor as there were no new anti-tuberculosis drugs and the second-line or reserve drugs used were less efficacious and more toxic then the first-line drugs. Since then, efforts are being made to optimize the therapy with first-line drugs to prevent occurrence of drug resistance and to develop new drugs or to find repurposed drugs for creating a potent second-line therapy. It is only in past about $5 \mathrm{y}$ that drugs like Bedaquiline and Delanamid have come up as new drugs effective against Mycobacterium tuberculosis. Very recently, WHO has used individual patient data from several countries to categorise and devise the drug therapy for multi drug resistance (MDR) and extreme drug resistance (XDR) in TB. Studies have been commissioned to understand the TB pharmacology and microbiology so as to improve the outcome of drug sensitive as well as drug resistant tuberculosis with minimal side-effects.

Sushil K. Kabra

skkabra@hotmail.com

1 Department of Pediatrics, Lady Hardinge Medical College and associated Kalawati Saran Children's Hospital, New Delhi, India

2 Department of Pediatrics, All India Institute of Medical Sciences, New Delhi 110029, India
The recent developments and current status of drug therapy is being published in this issue of Indian Journal of Pediatrics as the concluding part of this special two part symposium on advances in childhood tuberculosis. Singh V describes the evolution of the guidelines and strategies for the management of tuberculosis in children [1]. He underlines the essential change in understanding of TB epidemic in its current state which has led to development of newer technologies for early and fast diagnosis of drug resistance thus opening the path for a drug sensitivity based therapy for all cases. This is expected to decrease the occurrence of drug resistant tuberculosis (DRTB) by decreasing the risk of amplification of resistance. The article provides an overview of changes in treatment regimen for drug sensitive cases; doses of antituberculosis drugs and fixed dose combinations available under country's Revised National Tuberculosis Control Program (RNTCP).

Schaaf HS in his article on diagnosis and treatment of drug resistant TB has discussed practical approach in deciding which regimen to use in individual children with MDR TB [2]. He highlights that though the current regimes resulted generally in good outcomes but only $<5 \%$ of children with MDR TB are currently diagnosed and appropriately treated in time. This is worrisome as MDR TB has become a major global health concern even among children. Approximately 25,000 children develop multidrug resistant tuberculosis each year, but few of them are diagnosed and appropriately treated for MDR TB. Prof Schaaf highlights that the new diagnostic tools have improved our ability to diagnose children with bacteriologically confirmed TB earlier but still a majority of childhood TB cases are not bacteriologically confirmed. There is a need for a high index of suspicion and need for seeking detailed history of exposure to drug-resistant source cases and previous TB treatment to identify presumed MDR-TB cases. Early diagnosis of children with MDR-TB and getting them on to correct treatment regimens should now be an important aim of management.

Treatment for MDR TB is rapidly changing with the addition of new and repurposed drugs, the introduction of shorter regimens and the move towards injectable-free, all-oral MDR 
TB treatment regimens. Children have been neglected in the introduction of the new drugs, but drug dosing and safety studies are now being completed. Children with MDR TB are often treated with a regimen containing a fluoroquinolone, an aminoglycoside, ethionamide, cycloserine, pyrazinamide and ethambutol. In the absence of pediatric friendly tablets/ formulations, in most cases the adult tablets are either crushed or broken. This is likely to lead to inaccurate dosing. Very limited information is available on the pharmacokinetics of second-line anti-TB drugs in children with MDR TB, except for a few studies from South Africa and one from India. Drugs such as linezolid, clofazimine are also being considered for the treatment of MDR TB in children. In this issue of IJP, Ramchadran $G$ has reviewed the present limited knowledge of pharmacokinetics of second line drugs in children and raises the need for more studies to generate information on pharmacokinetics of ATT in children so that optimal, adequate dosing can be achieved [3].

Cruz and Starke, in their article on therapeutic drug monitoring discuss various aspects and need for drug level monitoring in selected group of children [4]. Most children tolerate the first-line drugs used to treat Mycobacterium tuberculosis (TB) very well. The most common adverse effect is gastrointestinal distress unrelated to hepatotoxicity; the latter is seen in less than $1 \%$ of children. Despite the infrequency of hepatotoxicity, the potential long-term impact of hepatic insufficiency dictates that all children receiving antimycobacterial therapy should be evaluated periodically by symptom screening and physical examination. Routine measurement of transaminases in previously healthy, symptomatic children is discouraged, as up to $40 \%$ of children will have transient, asymptomatic transaminase elevation that should not alter clinical management. Measurement of serum liver enzymes is reserved for children who develop symptoms and those with existing liver disease or taking other potentially hepatotoxic drugs. Caregivers and personnel distributing directly-observed therapy need to be cognizant of potential drug toxicities and have a clear understanding of what to do if a child develops symptoms. There are substantial inter-patient variations in serum antibiotic concentrations when the same milligram per kilogram dose is given to different children of varying ages and sizes, reflecting differences in drug absorption and metabolism. While these variations may not impact the outcome of previously healthy children with mild disease, outcomes for children with human immunodeficiency virus infection or severe disease can be worse if subtherapeutic drug concentrations are achieved. Therapeutic drug monitoring, wherein serum drug concentrations are used to optimize medication doses, should be considered for children with severe disease or if there is concern about alterations in drug absorption or metabolism.

Children suffer a huge and often an unrecognized burden of tuberculosis in endemic countries like India. Better data have improved the visibility of childhood TB, but the establishment of functional TB prevention and treatment programs for children remains challenging. Barriers to TB prevention include: 1) non-implementation of existing guidelines, 2) perceived inability to rule out active TB with fear of creating drug resistance and 3) limited local guidance on the use of preventive therapy after close contact with drug resistant TB. Barriers to TB treatment include: 1) diagnostic challenges in resourcelimited settings, 2) presentation to maternal and child health $(\mathrm{MCH})$ services with poor linkage to the TB control program and 3) limited local guidance on the treatment of children with likely drug resistant TB. Marais BJ in his article, provide an overview of newer drugs used for TB prevention and treatment in children [5]. He discusses new options for the treatment of latent TB infection (LTBI) and new or repurposed drugs used in the treatment of children with MDR TB. The background information provided describes the benefits, risks and feasibility of various treatment options, which should assist treatment decisions until updated World Health Organization (WHO) guidance becomes available.

Tuberculosis, once widely prevalent throughout the world, experienced falling incidence rates in early twentieth century in developed nations, even before the introduction of anti-TB drugs, attributed to improved hygiene and living conditions. Active TB may develop following fresh infection or activation of LTBI. For elimination of tuberculosis it is important to decrease the burden of LTBI in community to prevent reactivation in future. Now it is realized that identification and treatment of latent tuberculosis will go a long way in elimination of tuberculosis. Jain and Lodha, in their article on latent tuberculosis, discuss various aspects of LTBI including pathophysiology and diagnostic tests [6]. At present, there is no diagnostic test that can identify an individual with LTBI who will progress to develop active disease or remain asymptomatic. Therefore, it is unclear whom to treat. In the current scenario, treatment for LTBI is restricted to high risk groups which include under-5 y contacts of adults with pulmonary TB. Various regimens for treatment of LTBI are evolving and consist of isoniazid (INH) alone for 6-9 mo or combination of INH and rifampicin for 3-4 mo or once a week combination of rifapentine and INH for 3 mo. There is a need for research to identify LTBI, risk factors for progression of LTBI to active disease and a shorter regimen for treatment.

\section{Compliance with Ethical Standards}

Conflict of Interest None.

\section{References}

1. Singh V. Pediatric TB management under RNTCP: what and why? Indian J Pediatr. 2019. https://doi.org/10.1007/s12098-019-03001-7. 
2. Schaaf HS. Diagnosis and management of multidrug-resistant tuberculosis in children: a practical approach. Indian J Pediatr. 2019. https://doi.org/10.1007/s12098-018-02846-8.

3. Ramachandran G. Pharmacokinetics of second-line anti-tubercular drugs. Indian J Pediatr. 2019. https://doi.org/10.1007/s12098-01902923-6.

4. Cruz AT, Starke JR. Monitoring treatment of childhood tuberculosis and the role of therapeutic drug monitoring. Indian J Pediatr. 2019. https://doi.org/10.1007/s12098-019-02882-y.
5. Marais BJ. Newer drugs for tuberculosis prevention and treatment in children. Indian J Pediatr. 2019. https://doi.org/10.1007/s12098-01802854-8.

6. Jain A, Lodha R. Management of latent tuberculosis infection in children from developing countries. Indian J Pediatr. 2019. https://doi.org/10.1007/s12098-019-02861-3.

Publisher's Note Springer Nature remains neutral with regard to jurisdictional claims in published maps and institutional affiliations. 